\title{
Percutaneous Nephrolithotomy in Autosomal Dominant Polycystic Kidney Disease: Is it Different from Percutaneous Nephrolithotomy in Normal Kidney?
}

\author{
Vishwajeet Singh Rahul Janak Sinha Dheeraj Kumar Gupta \\ Department of Urology, King George Medical University, Lucknow, Uttar Pradesh, India
}

\section{Key Words}

Percutaneous nephrolithotomy • Nephrolithiasis •

Autosomal dominant polycystic kidney disease

\begin{abstract}
Objectives: Nephrolithiasis has been reported in $20-28 \%$ of patients, of whom $50 \%$ are symptomatic for stone disease and $20 \%$ require definite urologic intervention. The management of nephrolithiasis includes oral alkali dissolution therapy, extracorporeal shock wave lithotripsy and surgical treatment. In such patients, percutaneous nephrolithotomy (PNL) as a method of stone treatment has been reported in few cases with limited experience. The aim of this study is to present our experience of PNL in autosomal dominant polycystic kidney disease (ADPKD) and assessing the outcome results. Material and Methods: From 2002 to 2011, 22 patients (26 renal units) suffering from ADPKD with stone were managed by PNL. Demographic characteristics, operative parameters and postoperative complications were recorded and analysed. Result: The overall success rate of PNL was $82.1 \%$ and PNL with extracorporeal shock wave lithotripsy for clinically significant residual fragments was $92.85 \%$ respectively. The hematuria required blood transfusion $(n=9)$, postoperative fever due to cyst infection $(n=4)$ and paralytic ileus ( $n=3$ ) were recorded. Conclusion: The PNL in ADPKD is safe and effective but have more postoperative complications such as bleeding requiring transfusions, fever due to cyst infection and paralytic ileus. Copyright $\odot 2013$ S. Karger AG, Basel
\end{abstract}

\section{KARGER}

Fax +41613061234

E-Mail karger@karger.ch

www.karger.com
(C) 2013 S. Karger AG, Basel

$1015-9770 / 13 / 0071-0007 \$ 26.00 / 0$

Accessible online at:

www.karger.com/cu

\section{Introduction}

Autosomal dominant polycystic kidney disease (ADPKD) accounts for $8-10 \%$ of end stage renal disease. Most of the patients show a stable period of mild to moderate renal failure, which later on hastened by onset of hypertension, infection and nephrolithiasis [1]. Nephrolithiasis has been reported in $20-28 \%$ of patients, of whom $50 \%$ are symptomatic for stone disease and $20 \%$ require definite urologic intervention $[1,2]$. The causes of stone formation in ADPKD are due to both anatomic and metabolic factors. The management of nephrolithiasis includes oral alkali dissolution therapy, extracorporeal shock wave lithotripsy (ESWL) and surgical treatment [1-3]. There was time when open surgeries including nephrectomy were done for treatment of nephrolithiasis. In past few years these have largely been replaced by use of minimally invasive procedures such as ESWL and percutaneous nephrolithotomy (PNL). ESWL is a safe and effective method of treatment but less effective in large $(>2.5 \mathrm{~cm})$ stone [5-7]. PNL is an established method of stone treatment for large stone bulk in anatomic normal kidneys. In ADPKD patients, PNL as a method of stone treatment has been reported in few cases with limited experience [8-10]. Herein, we report our experience of PNL as a method to treat large stone bulk/ failed ESWL cases in 22 patients over 26 renal units. To the best of our knowledge the present series is the largest series of PNL in ADPKD patients in English literature. 


\section{Material and Methods}

In the retrospective study from 2002 to 2011, the records of 22 patients with 26 renal units of ADPKD underwent PNL were analysed. The clinical presentations of the patients are shown in table 1 . The kidney was palpable bilaterally in 18 patients and it was large renal lumps in 4 patients respectively. The complete hemogram, urinalysis, urine culture and sensitivity, renal function test, liver function test, coagulation profile and blood sugar level were performed. The renal and bladder ultrasound (fig. 1) and an X-ray kidney, ureter and bladder (KUB) were performed (fig. 2 ) in all cases. The intravenous urogram was done in 6 patients and contrast computer tomography (CT) scan abdomen in 8 cases respectively. The plain CT scan abdomen (fig. 3) or magnetic resonance urogram were performed in patients with renal failure. The patients presented with recurrent urinary tract infections were put on fluroquinolone till they became afebrile and urine culture became sterile. None of these 4 patients had infected renal cyst. A chest X-ray posteroanterior view, electrocardiogram and pulmonary function tests were performed to assess the fitness for anesthesia. In renal failure patients bilateral double J stenting was performed to relieve obstruction in 4 patients whose serum creatinine showed the downward trend but failed to reach baseline. Percutaneous nephrostomy was performed in 4 renal units to relieve the obstruction. The PNL was performed under combined spinal-epidural anesthesia in 10 and general anesthesia in 12 patients. The cystoscopy and ureteric catheterization was performed before the procedure. The patients were turned to prone position with bolsters under the chest wall for the full expansion of the lungs. The puncture was done by urologist under fluoroscopic guidance and the free efflux of injected saline from puncture needle was taken as correct puncture. The tract was dilated by Alken metallic dilator up to $26 \mathrm{~F} / 28 \mathrm{~F}$. The procedure was accomplished using $26 \mathrm{~F}$ (Richard Wolf) nephroscope. The stone was fragmented by pneumatic lithoclast (Swiss Lithoclast ${ }^{\circledR}$ ) and retrieved. In 4 patients the involvement was bilateral and both sides were operated simultaneously, but 2 patients required two-stage PNL. Single puncture and dilatation was done in 18 renal units and two-puncture and dilatations were done 8 renal units respectively. Single-stage procedure was performed in 12 patients and two-stage PNL in 10 patients respectively (table 2 ). A $6 \mathrm{~F}$ double $\mathrm{J}$ stent $/ 18 \mathrm{~F}$ nephrostomy tube was inserted at the end of the procedure. In patients with two-puncture and dilatations, 2 nephrostomy tubes were inserted. In 5 patients clinical significant residual fragments (CSRFs) ranging from $8 \mathrm{~mm}$ to $10 \mathrm{~mm}$ were seen for which ESWL was performed and stone clearance at the end of 3 months was achieved in 2 patients (table 2). A chest X-ray posteroanterior view was obtained in patients had supracostal puncture and dilatation. The renal ultrasound and an X-ray KUB were obtained on first postoperative day to check the stone residue, perirenal collections and hematoma. A hemogram, serum creatinine and urine cultures were sent on first postoperative day in each patient. The stone analysis was performed in 12 patients, showed calcium oxalate mixed with uric acid in 8 and pure calcium oxalate monohydrate in 4 patients respectively. The patients were followed-up with serum creatinine and renal ultrasound according to the standard follow-up protocol. A 24-hour urine examination for metabolic evaluation was performed in each patient. A nephrology consultation was obtained in patients had chronic renal failure and they were put on antihy- pertensives including angiotensin converting enzyme inhibitor or angiotensin receptor blockers.

\section{Results}

The mean patient age was 38 years (range 30-50 years). The right to left renal unit involvement ratio was 16:20 (4 patients had bilateral stones). The mean stone size was $28 \mathrm{~mm}$ (table 1). The mean operating time was 90 minutes (range 70-120 minutes). Single-stage PNL was done in 12 and two-stage in 10 patients. The CSRFs $(8-10 \mathrm{~mm})$ was observed in 5 patients treated by ESWL, 2 of these were stone free at 3 months (table 2). The overall success rate of PNL in this study was $82.1 \%$ and the result of PNL with ESWL was $92.85 \%$ respectively. The hematuria with hemodynamic instability was seen in 9 $(32.2 \%)$ patients required blood transfusion with mean packed red cell transfusion of 2.5 units ( $2-3$ units). The postoperative fever due to cyst infection observed in 4 patients (table 3 ). The fever did not respond and remained longer for which he was evaluated by renal ultrasound and infected cyst ( 2 infected cysts in each case) were aspirated. Pus culture showed E. coli sensitive to chloramphenicol and ciprofloxacin and they were treated for 12 weeks. The perirenal fluid collection persisted longer ( $>7$ days) in 3 patients which was aspirated by ultrasound guidance. In 4 patients there was significant $(>50$ $\mathrm{ml}$ ) perirenal hematoma following the procedure which was treated by conservatively and hematoma resolved in serial renal ultrasound (table 3 ). The renal failure was worsened in 3 patients (creatinine rise) who already had raised baseline creatinine but none required hemodialysis and responded by conservative measures including corrections of water and electrolytes disturbances. The hydrothorax developed in 2 patients required intercostal tube drainage, hemothorax in 1 patient treated by intercostal tube drainage and pneumothorax in 1 patient treated conservatively (table 3 ). The paralytic ileus was seen in 3 patients who had bilateral stones underwent bilateral PNL (table 3). The ileus was treated conservatively including cyst and perinephric fluid aspirations. The stone analysis was done in 12 patients showed the calcium oxalate mixed with uric acid in 8 and pure calcium oxalate monohydrate in 4 patients respectively. The 24-hour urine examination for the metabolic evaluation showed hypocitraturia in 10 , hyperuricosuria in 6 , hyperoxaluria in 5 and no metabolic abnormality in 3 patients respectively. The alkali dissolution therapy was given in all these patients. The patients are doing well in mean follow-up of 35 months. 
Table 1. Demographic characteristics of the patients

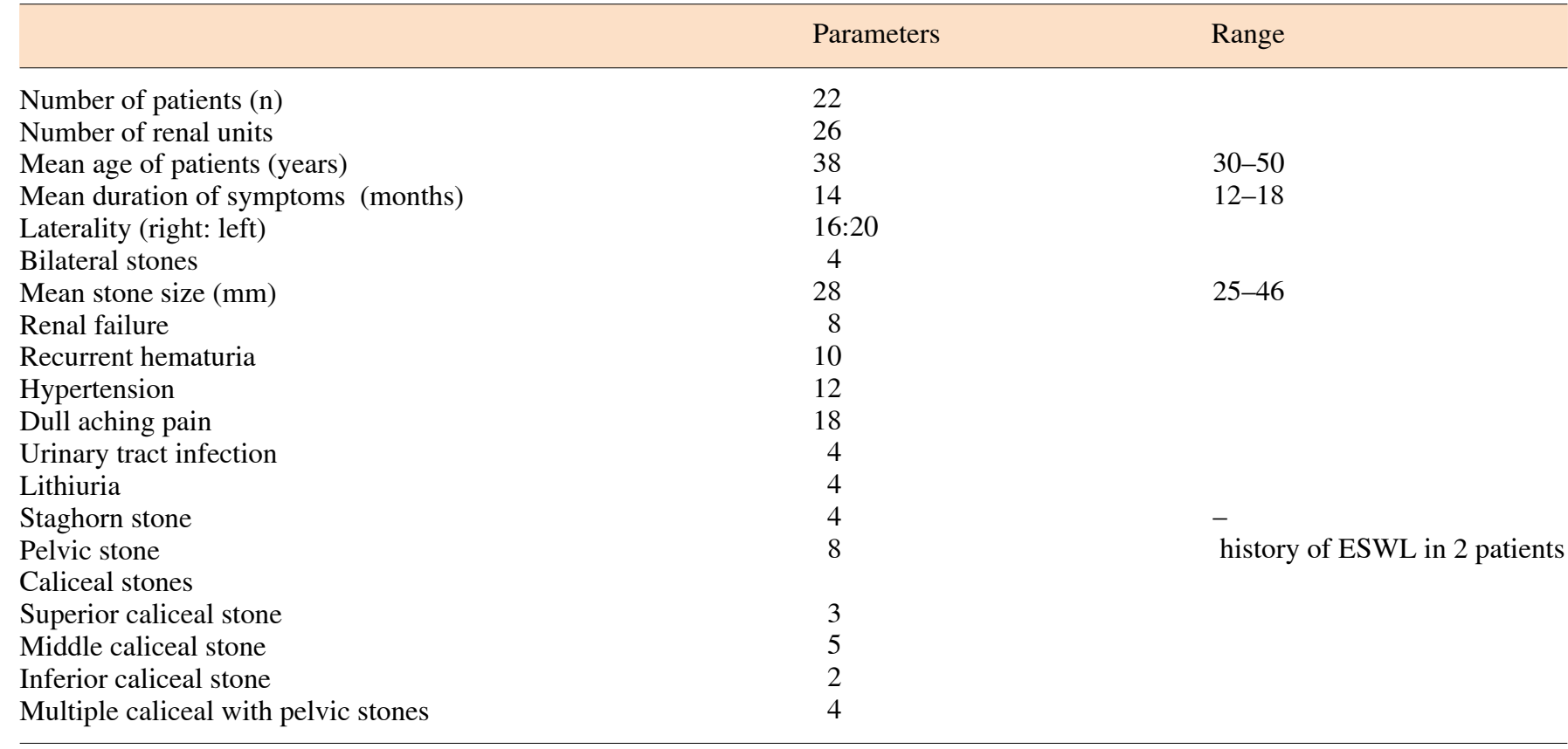

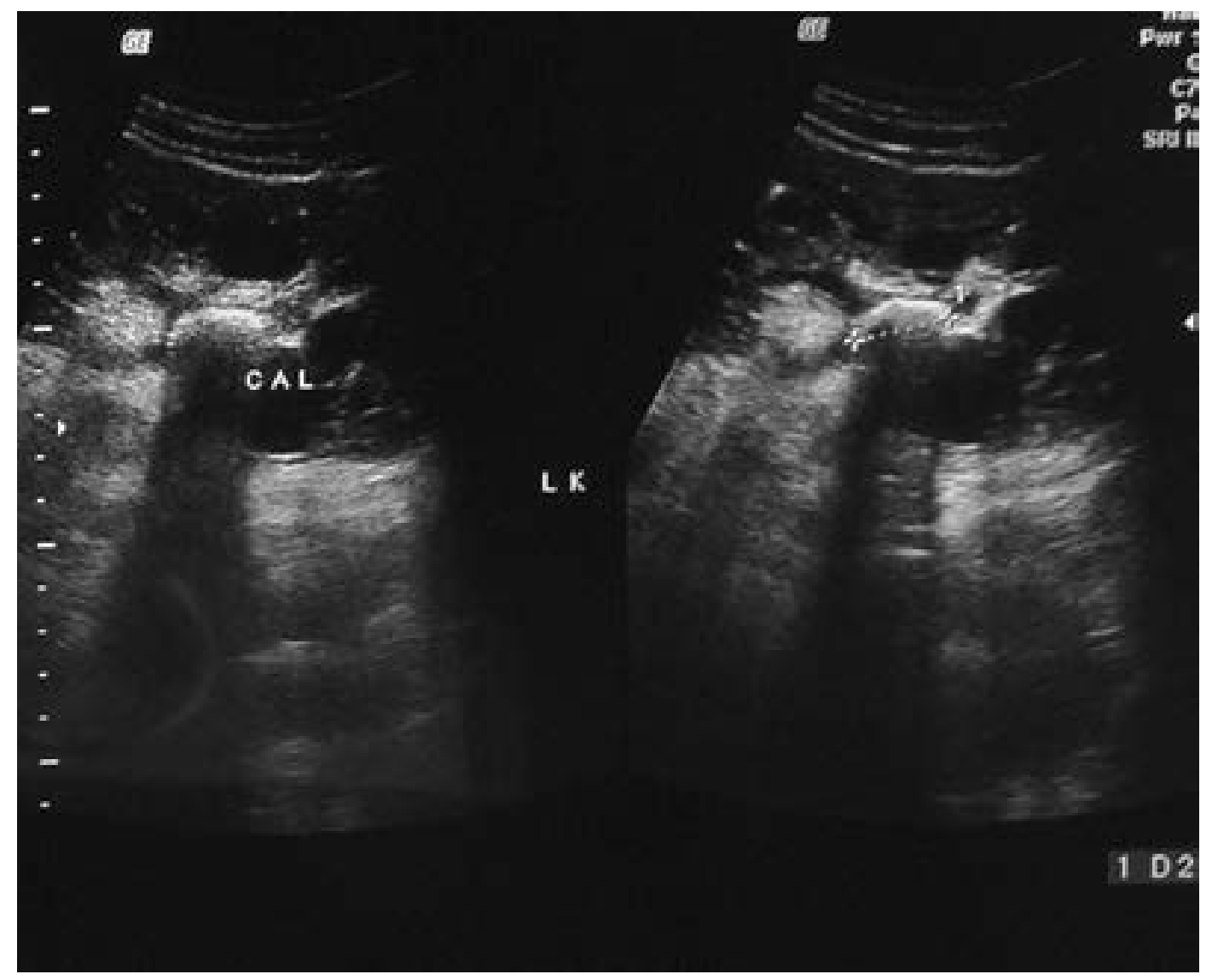

Fig. 1. Renal ultrasound showing left calculi with multiple cysts. 


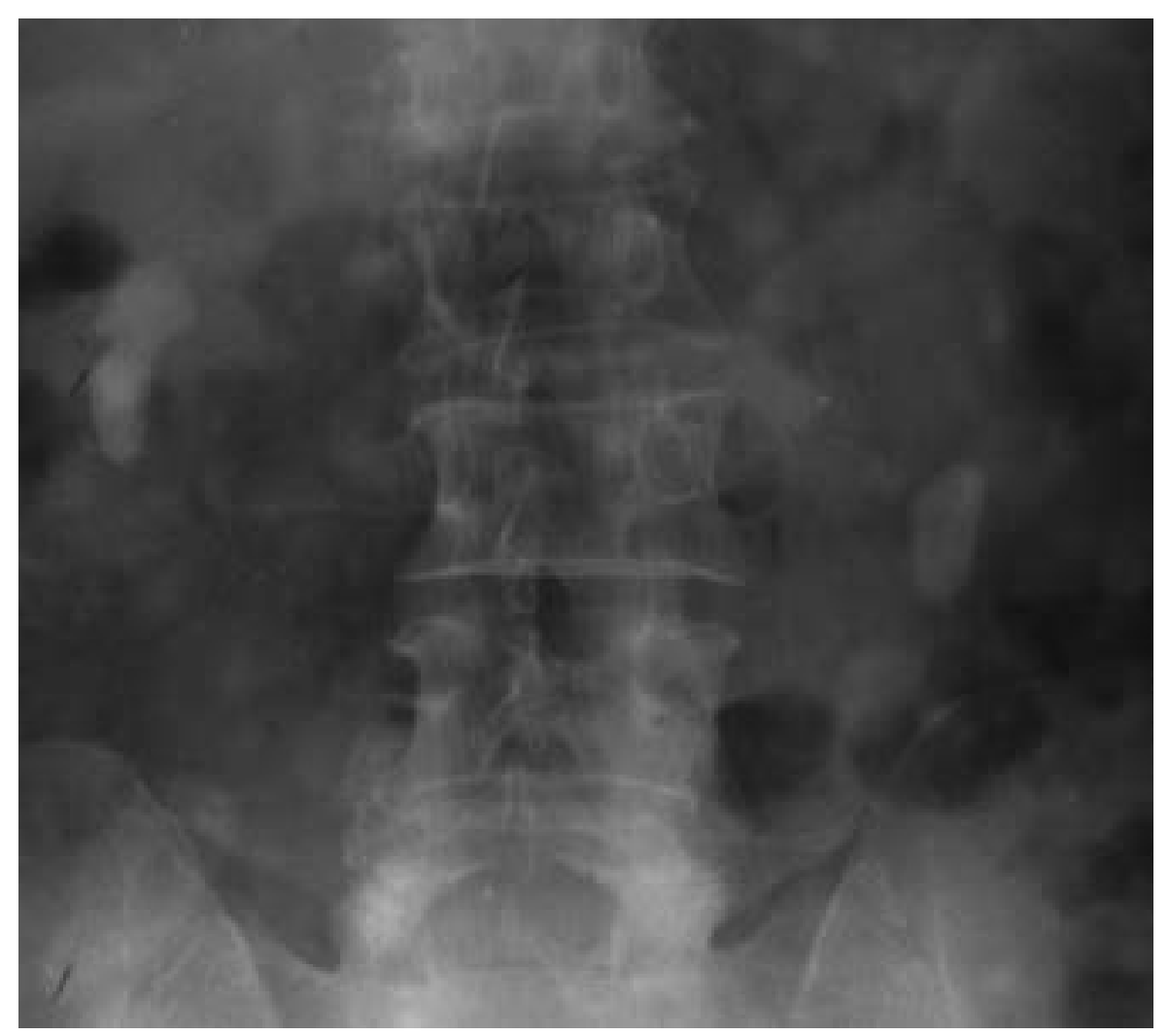

Fig. 2. KUB showing bilateral renal calculi.

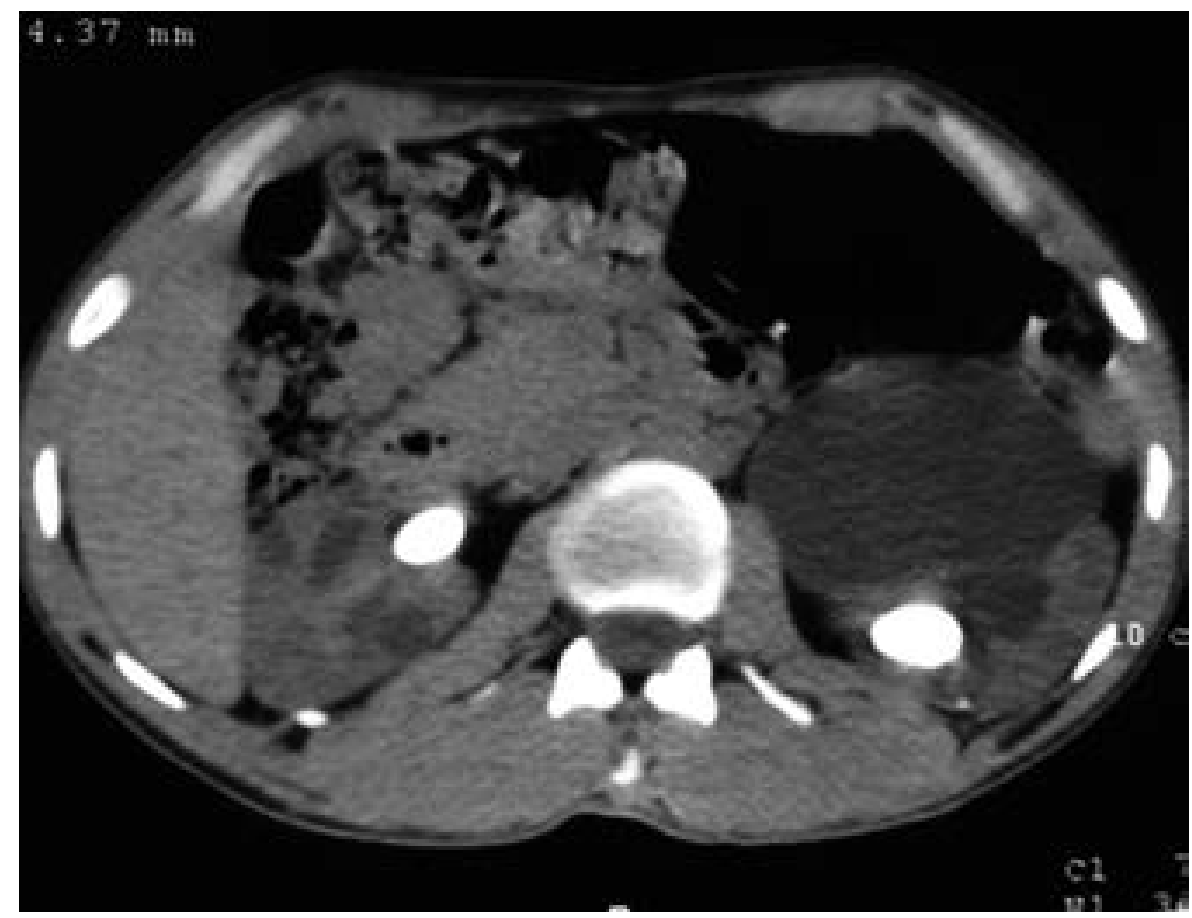

Fig. 3. Non-contrast CT scan abdomen showing bilateral polycystic Kidneys with calculi. 
Table 2. The operative parameters of the patients

\begin{tabular}{lll}
\hline & Reading & Range \\
\hline Operative time (minutes) & 90 & $70-120$ \\
Hematuria with hemodynamic instability requiring transfusion & 9 & - \\
Mean transfusion units for correction of hematocrit & 2.5 & $2-3$ \\
Mean fluoroscopy time (minutes) & 5 & $4-7$ \\
Mean irrigation volume (liter) & 12 & -14 \\
Intraoperative hypotension requiring resuscitation & 4 & - \\
but did not require termination of procedure & 12 & clearance in 3 months in 2 patients \\
Single-stage PNL & 10 & \\
Two-stage PNL & 5 & 12 \\
ESWL for CSRFs & 10 & \\
Initial superior caliceal puncture and dilatation & 4 & \\
Initial middle caliceal puncture and dilatation & & \\
Initial inferior caliceal puncture and dilatation & & \\
\hline
\end{tabular}

Table 3. The postoperative complications

\begin{tabular}{lc}
\hline Complications & Number of cases \\
\hline Hematuria requiring blood transfusion & 9 \\
Fever due to cyst infection & 4 \\
Perirenal hematoma collection & 4 \\
Hemothorax & 1 (required intercostal tube drainage) \\
Hydrothorax & 2 (required intercostal tube drainage) \\
Pneumothorax & 1 ( treated conservatively) \\
Paralytic ileus & 3 \\
Perirenal fluid collection treated by ultrasound guided aspiration & 2 \\
Worsening of pre-existing renal failure treated conservatively & 3
\end{tabular}

\section{Discussion}

ADPKD is an autosomal dominant disease which is an important cause of morbidity and mortality related to kidney disease. The important complications which occur due to this disease are the hypertension, renal failure, nephrolithiasis, hemorrhage and infections [1]. The nephrolithiasis occurs in about $8-36 \%$ of the patients which increases the morbidity and further accelerates the onset of renal failure [2]. There was a time when the open surgeries such as nephrectomy and nephrolithotomy were performed for the treatment of stones in ADPKD kidneys. The drawbacks of these procedures were the high morbidity rates. In recent years there has been consistent focus on the minimally invasive treatments such as ESWL and PNL [2-4]. The ESWL is indicated for the stones up to $20 \mathrm{~mm}$ with favorable outcome. For the larger stones and in failed cases of ESWL, PNL is the preferred modality. There are few papers and case reports on the PNL in ADPKD with limited number of patients and experience. The PNL in ADPKD create some difficulty such as distorted pelvicaliceal system leading to difficult puncture and dilatation, cyst puncture, cyst bleeding, cyst infection and associated parenchymal calcifications misleading to presence of stones under the fluoroscopy [1, 2, 4-8]. Umbreit et al. [9] found that PNL to be safe and effective in ADPKD with large stone burden despite the increased operative complexity, need 
of multiple punctures and repeat nephroscopy. In present study the puncture and dilatations were done under fluoroscopic guidance. Although the initial puncture was difficult because of distorted pelvicaliceal system but it was successful in all patients. Al-Kandari et al. [8] in a novel way used the diluted contrast material with methylthioninium chloride (blue dye) which was aspirated following puncture and it was taken as correct puncture. This was to avoid the cyst puncture and dilatation which is a genuine difficulty in ADPKD kidneys. In our study we did not use any dye rather following puncture and aspiration, if fluid was freely aspirated and comes out as jet efflux, it was taken as correct puncture and the tract was dilated subsequently. Baishya et al. [1] have advocated the ultrasound guided puncture and subsequent tract dilatation under fluoroscopic guidance. In our study we did not go for ultrasound guided puncture, because it could be difficult owing to the presence of multiple cysts that could have interfered with accurate localization of the compressed calices [8]. Bleeding remains a significant concern of PNL in ADPKD [1, 6, 8, 9]. The presence of multiple or large cysts, causing significant narrowing of the proper targeted calyx, could affect the approach and increase the risk of bleeding if forcible dilatation is tried. The compressive effect of cyst is more important than the size of cyst and their location to the target calyx. In such scenario, another favorable calyx then should be punctured to get entry in pelvicaliceal system and stones should be retrieved $[1,8,9]$. The bleeding leading to termination of the procedure was not observed in any of our case but the hematuria with hemodynamic instability was seen in 9 patients, managed conservatively. The PNL in ADPKD patients could be a staged procedure in which the puncture was done by a radiologist and later on the tract dilatation and stone retrieval by urologist $[8$, 9]. Intraoperatively the stone clearance can be enhanced by using the flexible nephroscope [8]. In our study we did not use the flexible nephroscope in any patients. The residual stone fragments can be managed by ESWL for smaller stones $[4,5]$. The ESWL was done in 5 patients of whom 2 were stone free in 3 months. The overall success of PNL in normally located kidneys is in the range of $84-96.5 \%$. The overall success rate in our study was $82.1 \%$ and the result of PNL with ESWL was $92.85 \%$ respectively which are comparable to existing literature $[16,18,19]$. The deterioration of renal function remains a concern of PNL in ADPKD patients. The renal trauma can cause loss of nephrons. In our patients the 3 patients had transient rise in serum creatinine. However the renal function in other patients were nearly stable in the post- operative period and it significantly improved in cases with obstructed systems, which was also similar to the observation by Al-Kandari et al. [8].

Bleeding is a major concern in PNL with an average hemoglobin drop ranging from 2.1 to $3.3 \mathrm{mg} / \mathrm{dl}$. As a result, $1-11 \%$ of patients overall and $2-53 \%$ of those underwent PNL for staghorn stones require blood transfusion. Most of the bleeding can be managed conservatively but it may require superselective angioembolization in $0.8 \%$ of patient. Rarely nephrectomy can be required to save the life [13-15]. The cystic kidneys predispose to hemorrahge is controversial. Al-Kandari et al. [8] have not found the ADPKD as a cause of bleeding which was similar to the observation by others. Idrizi et al. [6] have reported the cause of hematuria in ADPKD patients. They postulated that hematuria could be due to: 1) cyst rupture in pelvicaliceal system which can occur in making tract for PNL; 2) infection which can occur by irrigating fluid; 3 ) injury to the segmental renal arteries in making tract and; 4) injury by lithoclast probe for stone fragmentation. In our experience the hematuria leading to hypotension requiring blood transfusion was seen in $9(32.2 \%)$ which was similar to those reported in PNL series [13-15], but it was more in comparison to the incidence of hematuria in our previous study on PNL in normal kidneys [16]. All our patients were managed by conservative measures including multiple transfusions but none required angioembolization.

Cyst infections could be a cause of fever in ADPKD kidneys. The urine culture remains sterile in most of the cases. The diagnosis is done based clinically on the development of discrete new palpable areas of renal tenderness. The imaging studies with renal ultrasonography or CT scan give valuable information $[6,17]$. The refractory nature of cyst infection has been shown to be largely due to poor penetration of commonly used antibiotics in cyst fluid. Diffusion of antibiotics across the cyst wall is dependent upon the lipid solubility. Lipophilic antibiotics such as trimethoprim, fluoroquinolone, chloramphenicol and metronidazole rapidly achieve high intracystic concentration [17]. The postoperative fever due to cyst infection was observed in 4 patients who were not responding to cephalosporins. A renal ultrasound revealed the infected cysts which were aspirated and pus culture showed $E$. coli and they were treated initially by chloramphenicol and metronidazole. Later oral ciprofloxacin was continued till 12 weeks.

The surgical intervention in ADPKD patients can lead to paralytic ileus. The cause of the ileus could be secondary to accumulation of cystic fluid and blood clots 
in retroperitoneum. The irrigation fluid extravasation and infection could be the other causes. In present study the ileus was observed in 3 patients and all these had bilateral PNL. They had renal failure. The uremia, infection, fluid and electrolyte imbalance, cystic fluid, retroperitoneal blood and irrigation fluid extravasation all would have caused the paralytic ileus. In 2 patients the multiple cysts and retroperitoneal fluid were aspirated which led to recovery from ileus, similarly reported by others $[20,21]$.

\section{Conclusion}

The PNL in ADPKD patients is safe and effective procedure but the procedure is more associated with hematuria requiring blood transfusion, postoperative fever due to cyst infection and paralytic ileus.

\section{References}

1 Baishya R, Dhawan DR, Kurien A, Ganpule A, Sabnis RB, Desai MR: Management of nephrolithiasis in autosomal dominant polycystic kidney disease-A single centre experience. Urol Ann 2012;4:29-33.

2 Grampsas SA, Chandhoke PS, Fan J, Glass MA, Townsend R, Johnson AM, Gabow P: Anatomic and metabolic risk factors for nephrolithiasis in patients with autosomal dominant polycystic kidney disease. Am J Kidney Dis 2000;36:53-57.

- 3 Firinci F, Soylu A, Demir BK, Turkmen M, Kavukcu S: An 11-year-old child with autosomal dominant polycystic kidney disease who presented with nephrolithiasis. Case Report Med 2012;2012:428749.

-4 Delakas D, Daskalopoulos G, Cranidis A: Extracorporeal shock wave lithotripsy for urinary calculi in autosomal dominant polycystic kidney disease. J Endourol 1997;11:167-170.

5 Nishiura JL, Neves RF, Eloi SR, Cintra SM, Ajzen SA, Heilberg IP: Evaluation of nephrolithiasis in autosomal dominant polycystic kidney disease patients. Clin J Am Soc Nephrol 2009;4:838-844.

6 Idrizi A, Barbullushi M, Petrela E, Kodra S, Koroshi A, Thereska N: The influence of renal manifestations to the progression of autosomal dominant polycystic kidney disease. Hippokratia 2009;13:161-164.

7 Ng CS, Yost A, Streem SB: Nephrolithiasis associated with autosomal dominant polycystic kidney disease: contemporary urological management. J Urol 2000;163:726-729.
8 Al-Kandari AM, Shoma AM, Eraky I, El-Kenawy MR, Al-Eezi H, El-Kappany HA: Percutaneous nephrolithotomy for management of upper urinary tract calculi in patients with autosomal dominant polycystic kidney disease. Urology 2009;74:273-277.

9 Umbreit EC, Childs MA, Patterson DE, Torres VE, LeRoy AJ, Gettman MT: Percutaneous nephrolithotomy for large or multiple upper tract calculi and autosomal dominant polycystic kidney disease. J Urol 2010;183: 183-187.

10 Srivastava A, Bansal R, Srivastava A, Chaturvedi S, Ranjan P, Ansari MS, Yadav A, Kapoor R: Percutaneous nephrolithotomy in polycystic kidney disease: is it safe and effective? Int Urol Nephrol 2012;44:725-730.

11 Torres VE, Wilson DM, Hattery RR, Segura JW: Renal stone disease in autosomal dominant polycystic kidney disease. Am J Kidney Dis 1993;22:513-519.

12 Levine E, Grantham JJ: The role of computed tomography in the evaluation of adult polycystic kidney disease. Am J Kidney Dis 1981;1:99-105.

13 Kukreja R, Desai M, Patel S, Bapat S, Desai M: Factors affecting blood loss during percutaneous nephrolithotomy: prospective study. J Endourol 2004;18:715-722.

14 Srivastava A, Singh KJ, Suri A, Dubey D, Kumar A, Kapoor R, Mandhani A, Jain S: Vascular complications after percutaneous nephrolithotomy: are there any predictive factors? Urology 2005;66:38-40.
15 Kessaris DN, Bellman GC, Pardalidis NP, Smith AG: Management of hemorrhage after percutaneous renal surgery. J Urol 1995;153: 604-608.

16 Singh V, Sinha RJ, Sankhwar SN, Malik A: A prospective randomized study comparing percutaneous nephrolithotomy under combined spinal-epidural anesthesia with percutaneous nephrolithotomy under general anesthesia. Urol Int 2011;87:293-298.

17 Bennett WM, Elzinga L, Pulliam JP, Rashad AL, Barry JM: Cyst fluid antibiotic concentrations in autosomal dominant polycystic kidney disease. Am J Kidney Dis 1985;6: 400-404.

18 Shoma AM, Eraky I, El-Kenawy MR, El-Kappany HA: Percutaneous nephrolithotomy in prone and supine position: technical aspects and functional outcome compared with the prone technique. Urology 2002;60:388-392.

19 Osman M, Wendt-Nordahl G, Heger K, Michel MS, Alken P, Knoll T: Percutaneous nephrolithotomy with ultrasonography-guided renal access: experience from 300 cases. BJU Int 2005;96:875-878.

20 Kakinoki K, Noda Y, Takaeda M, Kubo M, Mizuhashi K, Miyamoto I, Nishida T, Takemori Y: Intestinal obstruction in autosomal polycystic kidney disease. Intern Med 2002; 41:441-444.

21 Kato T, Ubara Y, Tagami T, Sawa N, Hoshino J, Suwabe T, Yamagata T, Katori H, Takemoto F, Hara S, Matoba S, Takaichi K: Autosomal polycystic kidney disease showing rupture of a lateral ventral hernia following paralytic ileus. Intern Med 2005;44:311-314. 